\title{
Availability analysis of resilient geometric routing on Internet topology
}

\author{
Sahel Sahhaf*, Wouter Tavernier*, Didier Colle*, Mario Pickavet* and Piet Demeester* \\ *Department of Information Technology (INTEC), Ghent University, iMinds \\ Gaston Crommenlaan 8, 9050 Gent, Belgium \\ Email:\{sahel.sahhaf, wouter.tavernier, didier.colle, mario.pickavet, piet.demeester\}@intec.ugent.be
}

\begin{abstract}
Scalable routing schemes for large-scale networks, especially future Internet, are required. Geometric routing scheme is a promising candidate to solve the scalability issue of routing tables in conventional IP routing based on longest prefix matching. In this scheme, network nodes are assigned virtual coordinates and packets are forwarded towards their intended destination following a distance-decreasing policy. Dynamics in the network such as node/link failures might affect this forwarding and lead packets to a dead end. We proposed recovery techniques in geometric routing to deliver packets to the destination in case of failures. In this paper, we perform an analysis on the availability of the proposed protection techniques on the Internet graph. The routing scheme over optical transport network is considered and the reliability data of physical components and a known network availability model are used. This evaluation is compared with the shortest cycle scheme which finds two node disjoint paths between every source and destination in the topology and also with geometric routing with no protection. The results show that the proposed scheme performs reasonably well compared to the shortest cycle scheme and significantly enhances the availability compared to geometric routing without any protection.
\end{abstract}

\section{INTRODUCTION}

Due to ever increasing throughput demands, IP prefix lookup is becoming a bottleneck in future Internet. Higher performance, capacity and forwarding rates are required in order to meet the future demands. The growth of the size of the routing tables is another issue which limits the scalability of the current IP-based routing protocols. BGP is the protocol used to exchange routing and reachability information between Autonomous Systems (AS) in the Internet ([1] reports more than $400 \mathrm{~K}$ FIB entries in current BGP routers). Geometric routing has been proposed to solve the issue of scalability in routing tables and can be considered as an alternative to BGP to route between ASes (Inter-AS).

In geometric routing, nodes are assigned virtual coordinates and the forwarding is based on a distance-decreasing policy [2]. This means that upon arrival of a packet in a node, the distance of every neighboring node to the destination of the packet is calculated and the one which decreases this distance the most is selected as the next hop. Repeatedly applying this policy will lead the packets to their intended destination. As in every step, the neighbor with the most decreasing distance is selected, the routing/forwarding is referred as greedy.

A problem with greedy forwarding is that packets might reach a local minimum (dead end, void). This means that the current node is closer to the destination than any of its neighbors. Greedy embeddings are proposed to solve this issue [3]. Greedy embeddings map network nodes to coordinates in such a way that for every node there is always a distance decreasing neighbor towards any other node in the network. Greedy routing based on these embeddings guarantees the delivery of the packets to every destination. In [4], we proposed a simple but promising greedy embedding based on a spanning tree of the network. In this scheme, every node is assigned a label (coordinate) indicating the path from the root of the tree to the node.

As the embedding is derived from a spanning tree of the network, a change in the connectivity of the tree (component failures) might affect the embedding and might lead the packets to local minima. Therefore, recovery techniques are required to provide resiliency against failures in the network. In [4] and [5], we proposed protection techniques in geometric routing for link/node failures in the network. These works showed the scalability of the proposed schemes in terms of resources. As availability is an important performance assessment factor for recovery schemes, the goal of this paper is to evaluate the connection availability of the proposed single-failure resilient geometric routing on a large graph such as the ASlevel topology of the existing Internet (CAIDA [6]). In this evaluation, geometric routing over optical transport network is considered and the reliability characteristics of physical components and a well-known availability model are used. In order to have a realistic evaluation, a multi-layer model is used in which recovery in different layers is considered. Therefore, we evaluate the connection availability of geometric routing as a network layer scheme with the assumption that links are protected in lower layers. Having a fair comparison with BGP is challenging because different factors such as routing policies and AS business relationships affect the selection of the paths. Therefore, we compare the availability of the proposed scheme with shortest cycle scheme in which two node disjoint paths between every source and destination in the network are constructed. In this scheme, in case the working path between two nodes is not available, the second node disjoint shortest path is used as the protection path. To the best of our knowledge, this is one of the first studies related to connection availability of geometric routing on the Internet topology.

This evaluation gives an overview on: i) how well the proposed recovery scheme performs compared to the node disjoint shortest path alternative and ii) what is the maximum and minimum connection availability that can be obtained using the model proposed on the Internet topology. These studies are essential especially for services with high availability demands.

The rest of the paper is organized as follows. In the next 
section, work related to availability analysis in network, geometric routing and recovery techniques are described. A short description of the used greedy embedding and the protection techniques is provided in Section III. Section IV explains basic concepts related to reliability assessment. Section V presents the evaluation of connection availability for different schemes of single-failure resilient geometric routing, shortest cycle and geometric routing with no protection. Future work is discussed in Section VI and finally Section VII concludes the paper.

\section{RELATED WORK}

Availability analysis especially in wavelength division multiplexing (WDM) optical networks has received great attention in recent years. Many works have proposed analytical models for evaluating the connection availability in these networks for single link failure scenarios [7], [8]. The main observation in [7] was that a mesh network with single link failure restorability is robust under dual-failure events. The authors defined equivalent unavailability for a link which means that the link is considered available if it is physically working or it is physically down but transparently replaced by a restoration path between its end nodes. This is similar to the multi-layer model we consider in the connection availability evaluation in this paper. While evaluating the availability of routing scheme in network layer, we assume that links are protected in lower layers. In [8], different protection schemes, 1:1, 1:N and M:N are considered. The focus of the paper is on path protection strategy in which a backup path for each working path on an end-to-end basis is provided. This is different from our work as nodes are recovered locally and failing links are bypassed by routing towards a hub node. In [9], the reliability analysis on two link-disjoint paths in mesh network is studied. Using two disjoint paths, $100 \%$ protection against a single failure is provided and authors try to maximize the reliability regardless of the number of link failures occurring on the network. It is proved that this problem is NP-complete and heuristic algorithms are proposed to find two link-disjoint paths with maximum reliability. In this paper, we compare the connection availability of the proposed schemes for geometric routing with such an scheme in which two disjoint paths for every source and destination in the topology are calculated.

In other works such as [10] analytical model for dual link failure is proposed. In [11], authors proposed a hybrid scheme to achieve high connection availability with low backup resources in double link failure scenario. The hybrid scheme is based on backup re-provisioning, path restoration and 1:1 dedicated path protection. They use ILP models in their proposed scheme. In [12], a model for availability evaluation of protected optical connections in WDM networks employing M:N dedicated protection is proposed. The same authors proposed an analytical model for availability evaluation of WDM network with shared-link connections under multiple link failures in [13]. The effect of topology properties on connection availability in Generalized Multiprotocol Label Switching (GMPLS) over optical transport networks is studied in [14].

As geometric routing scheme suffers from packets reaching local minima, many works have proposed greedy embeddings in different metric spaces to avoid this issue. In [15] and [16], authors proposed greedy embeddings in the hyperbolic plane.

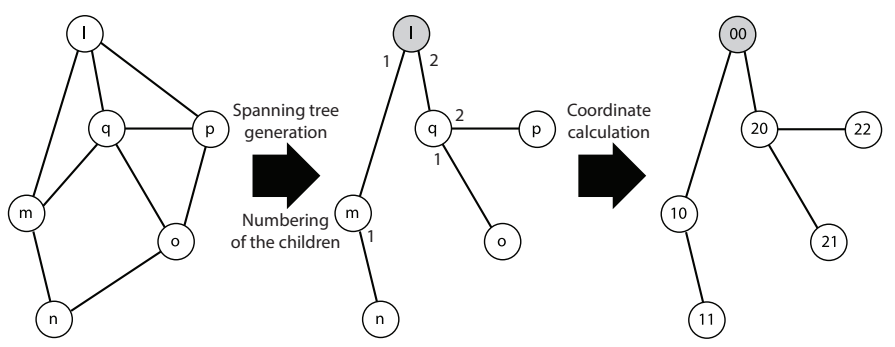

Fig. 1. Steps for tree-based greedy embedding.

Flury et al. proposed greedy embedding in Euclidean space [17]. We proposed a greedy embedding based on a spanning tree of the network in [4]. Dynamics in the topology such as link/node failure might affect the greedy embeddings causing the packets reaching local minima. Face routing techniques pass the void by routing around this area and greedy routing is resumed from the moment that a closer node to the destination is reached [18]. The issue with these type of techniques is that the graph should be planar/planarized which might not be feasible for every graph. Authors in [19] proposed a dynamic greedy embedding to deal with node additions to the network topology. However, in order to deal with network failures a path trace is maintained in packets in order to avoid loops which imposes a large overhead to packets headers [4]. We proposed different protection techniques for link/node failures which avoid coordinate re-calculations and are based on greedy forwarding [4], [5]. The proposed schemes are scalable with low overhead to the network nodes and packets headers.

In this paper, we consider the tree-based greedy embedding as proposed in [4] and evaluate connection availability of geometric routing with the proposed protection schemes in [4] and [5] over optical transport network in the Internet topology.

\section{GREEDY GEOMETRIC ROUTING}

In this section, we briefly explain the tree-based greedy embedding and the protection techniques on which the availability analysis is performed. For more details on the schemes, we refer the interested readers to [4] and [5].

\section{A. Tree-based greedy embedding}

Rather than relying on complex (e.g. hyperbolic) geometry, in [4] we used tree-based coordinates for greedy forwarding. This will reduce the computational overhead and complexity of the overall scheme. Steps for calculating the labeling based on a spanning tree of the network are as follows:

1) First a rooted spanning tree of the network is generated.

2) The root node sets its coordinate vector (CV) to zero.

3) Each node numbers its children from 1 to $d$ ( $d$ is the number of children).

4) A node can calculate the coordinates of its children by adding the number assigned to each child after the last non-zero coordinate in its own $\mathrm{CV}$.

Figure 1 depicts an example for this embedding. Once every node has its deduced CV using the above procedure, packets can be forwarded towards neighboring nodes which 

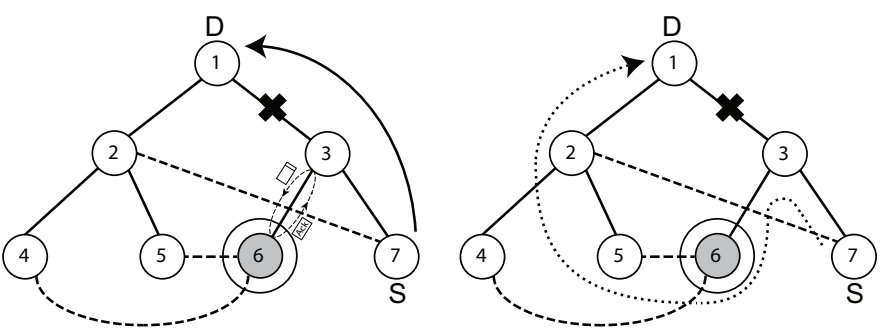

Fig. 2. Example for upward failure scenario.

(maximally) reduce the distance towards the $\mathrm{CV}$ of the destination node mentioned in the received packet. In this context, tree-distance is used as the metric which is the hop count on the tree between two nodes.

\section{B. Single failure protection techniques}

In [5], we proposed protection techniques for geometric routing which are only used for tree edge failures. The reason is that in greedy routing based on a spanning tree, in case of a shortcut ${ }^{1}$ failure, there is still a distance-decreasing path available via the tree. However, a tree edge failure might affect the greedy embedding causing the packets to reach a local minimum.

In this scheme, we distinguish between upward and downward failures. In upward failures, packets should have passed the failing link to get closer to the root of the tree while in downward failures, packets go deeper in the tree.

For every tree edge attached to a node, a search is performed in order to find an intermediate node (hub node) from which greedy routing to the destination is possible (considering that edge to be failing). In a downward failure scenario, we look for a hub node which has a shortcut to the subtree below the failing link. In an upward failure scenario, it is enough that the hub node has a shortcut to a node out of the subtree below the failing link. The forwarding process in case of a failure is as follows:

1) Greedy route from failure detecting node to hub node.

2) Take the shortcut to go to the desired subtree.

3) Continue greedy routing to the destination on the tree.

Figures 2 and 3 depict examples for the two explained failure scenarios. The primary greedy path is depicted with solid line on the left and the secondary path after recovery is depicted with dot line on the right. The corresponding hub node for the depicted failing tree edge is colored grey.

We proposed a protection technique for node failure in [4]. In this scheme, every node finds disjoint backup paths to the nodes two hops away. In case of a node failure, a disjoint backup path is used to bypass the failing node and greedy routing is resumed once the two-hops away node is reached.

We refer the interested readers to [4] and [5] for more details on the exchanged messages, augmentation to the packet headers and the overhead added to the network nodes.

\footnotetext{
${ }^{1} \mathrm{~A}$ link which is not in the spanning tree of the network.
}

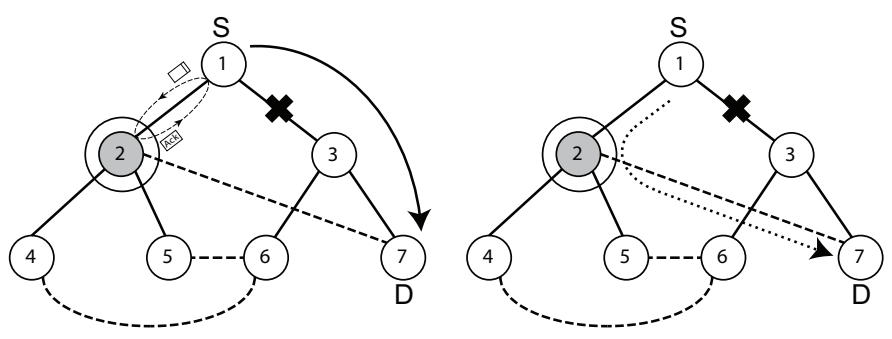

Fig. 3. Example for downward failure scenario.

\section{PERFORMANCE PARAMETERS}

In this section, we explain the reliability performance parameters which are used for the evaluation of the protection techniques for single failure in geometric routing.

\section{A. Component availability}

Availability of a component is defined as the probability that the component is functional at any arbitrary moment. It can be expressed by the components mean time to repair (MTTR) and the mean time between failures (MTBF). MTTR is defined as the required time for the restoration of the component and MTBF is the time between two consecutive failures of the component [20]. The availability $A$ is:

$$
A=1-\frac{M T T R}{M T B F}
$$

\section{B. Protected/Unprotected connection availability}

The availability of a connection in a network is calculated using the availability of the components (nodes/links) in the network. In an unprotected connection, all the nodes and links/lines along the path of the connection should be available in order to have availability for the connection. Therefore, the availability is defined as:

$$
A_{\text {unprotected }}=\prod A_{i}
$$

$A_{i}$ is the availability of the $i$ th component (node or link) along the path.

As mentioned earlier, recovery schemes enhance the availability of the connection. A protected connection is available if the working path or the protection path of the connection is available.

$$
A_{\text {protected }}=A_{w}+A_{w}^{\prime} A_{p}
$$

$A_{w}$ and $A_{w}^{\prime}$ are the availability and unavailability of the working path respectively. $A_{p}$ is the availability of the protection path. This formula can be extended for the availability of a system, $A_{\text {system, }}$, with more than one connection and considering single failure:

$$
A_{\text {system }}=\prod_{i=1}^{M} A_{w_{i}}+\sum_{j=1}^{M}\left(\prod_{k=1 ; k \neq j}^{M} A_{w_{k}}\right) A_{w_{j}}^{\prime} A_{p_{j}}
$$

$M$ is the number of working connections in the system. 


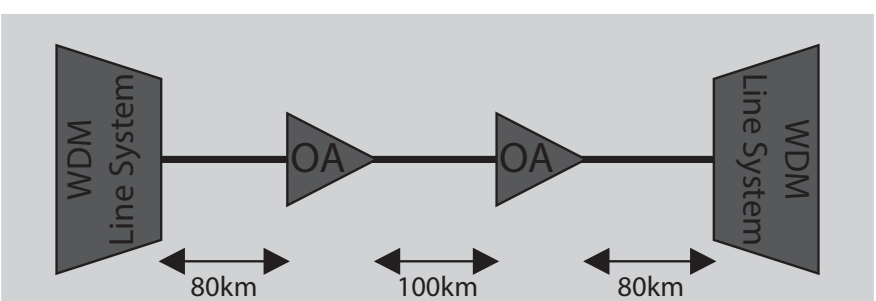

Fig. 4. Example of a bidirectional line [20].

\section{Network availability model}

In the network availability model considered in this paper, a node is composed of one Optical cross connect (OXC). A bidirectional line which connects two optical nodes is composed of a series of components such as pieces of physical cable, a number of optical amplifiers (OA) and a line system at each side of the line (Figure 4). This line is available only if all the components are available. Assuming statistically independent failures, the availability of a bidirectional line is defined as:

$$
A_{\text {line }}=A_{\text {cable }} \times A_{O A}^{N} \times A_{\text {line-system }}^{2}
$$

$N$ is the number of OAs which is dependent on the length of the line (assuming an OA every $100 \mathrm{Km}$ ) [20].

The MTTR and MTBF of physical components reported in [20] are used in formula (1) to obtain the availability of single components in the network.

We evaluate the connection availability of geometric routing over optical transport network for every possible sourcedestination pair in the topology based on the reliability characteristics of the physical components and the connection model in formula (3). In order to obtain a more realistic evaluation, we consider a multi-layer model. This means that although the proposed protection scheme is provided for network layer (L3), in the availability evaluation of the connections, links in the topology are considered to be protected in lower layers (L2/L1).

Note that in the proposed protection scheme, in a connection, depending on the link/node which is failing, different backup paths might be used. Therefore, in order to evaluate the availability of a connection, every possible single failure along the connection path is considered and the availability of the corresponding backup path is evaluated and the sum of all represents the protected connection availability. Consider Figure 5 as a case study. In this example, only link failure is considered. Each of the graphs in this figure depicts a single link failure scenario along the connection 7-3-1 and the corresponding protection path is illustrated by a dot arrow. The availability of the connection 7-1 is calculated as follows:

$$
\begin{aligned}
A_{\text {connection } 7-1}= & A_{7-3} A_{3-1}+A_{7-3}^{\prime} A_{7-2} A_{2-1}+ \\
& A_{3-1}^{\prime} A_{7-3} A_{3-6} A_{6-5} A_{5-2} A_{2-1}
\end{aligned}
$$

\section{RELIABILITY PERFORMANCE EVALUATION OF CONNECTIONS}

In this section, we evaluate the connection availability of the Internet topology for different schemes: i) tree-based geometric routing with no protection and ii) tree-based geometric
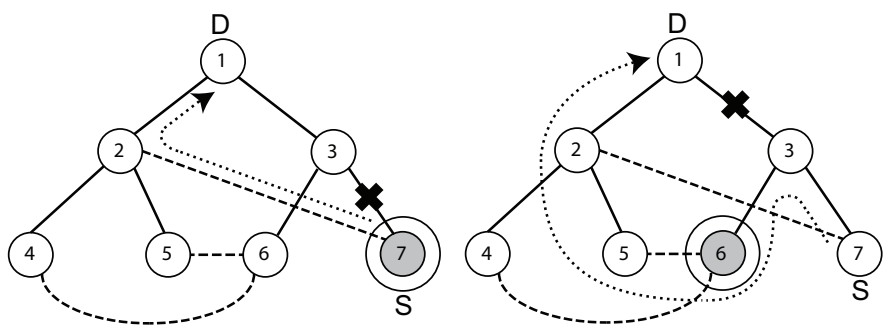

Fig. 5. Example for evaluation of connection availability.

routing with single link/node failure protection technique. In all schemes, we assume that links are protected in lower layers (L2/L1). Having a fair comparison with BGP is a challenging task because in BGP different factors such as AS relationships and routing policies affect the path selection. Therefore, we compare the schemes with another alternative in which the shortest cycle between every source and destination is calculated. In this scheme, in case of the unavailability of the working path, the second node disjoint shortest path is used as the protection path.

First, we explain our simulation methodology and how we modeled the Internet topology and then we report the numerical results.

\section{A. Methodology for simulation approach}

The general steps in our simulation are as follows:

1) Given a topology, the tree-based coordinates as explained in Section III are assigned to the network nodes.

2) The hub node corresponding to every tree edge and the disjoint backup paths for nodes are calculated as explained in Section III.

For every possible connection in the network (every possible source-destination pair):

1) The path produced by greedy geometric routing is determined.

2) The availability of the unprotected connection is calculated based on formula (2).

3) Components (link/node) along the path are considered failing, one at a time, the protecting path corresponding to that failure is calculated based on the proposed protection technique and the availability of the connection is calculated as explained in Section IV.

In the availability evaluation of the scheme based on shortest cycle, as for any failure in the working path, the same backup path is used, we directly apply formula (3).

\section{B. Internet topology}

As mentioned earlier, geometric routing is considered as an alternative to BGP to route between ASes in the Internet (interAS routing). Therefore, we perform our availability analysis on an AS-level graph of CAIDA which represents the topology of the Internet at the level of ASes. Figure 6 visualizes CAIDA's AS-level Internet graph in January 2013. Due to resource 


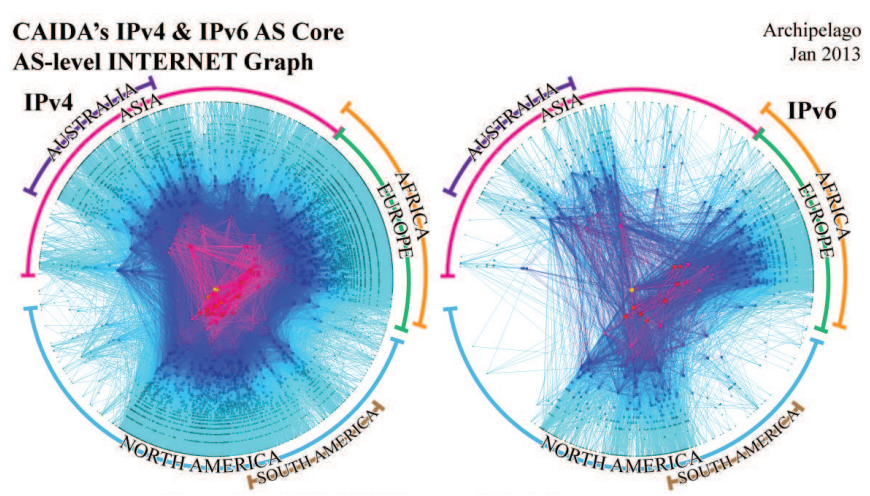

Fig. 6. CAIDA's AS-level Internet graph. http://www.caida.org

limitation in the simulation environment, we select the CAIDA network which consists out of 16301 ASes and 27646 links [6]. For this network, the average shortest path is 3.77 , the minimum node degree is 1 and the maximum node degree is 2331.

One of the major challenges in this analysis is to achieve a network model based on the existing data for the Internet topology. In the following, we explain how these challenges are tackled and what assumptions are made.

In CAIDA AS-level graph, nodes represent ASes and links represent peering relationships. No more data regarding the geolocations and the distances between different ASes is provided. In order to evaluate the connection availability on the CAIDA topology, we require some estimation on the physical distance between adjacent nodes in the network (required for line availability evaluation in formula (5)). To this end, we used two different data bases ${ }^{2}$ : i) GeoLite ASN which provides the IP addresses to AS numbers mapping and ii) GeoLite City which provides IP addresses to geolocations mapping. Using these databases, we extract the approximate location of each node in CAIDA topology. Assuming that each AS consists of one border router (due to lack of more information), the extracted locations represent the geolocation of such routers. The border router of an AS is assumed to be directly connected to the border routers of adjacent ASes. Therefore, the physical distance between connected routers is calculated based on their geolocations. These border routers perform geometric routing to route between ASes.

The first challenge was that the databases could not provide geographic location for almost $10 \%$ of the nodes in the network. Therefore, in order to estimate the location of them we used the locations of the adjacent nodes. Based on the locations of the neighboring nodes, the geolocation of the center point is calculated and is assigned to the node without any location. This way, we could estimate the locations of the majority of the nodes with missing locations.

The next challenge was that this process could not be used for the leaf nodes (nodes with only one neighbor). Therefore, in such scenarios we used another process to complete our model.

Consider a leaf node with missing location which has a

\footnotetext{
${ }^{2}$ http://dev.maxmind.com/geoip/legacy/geolite/
}

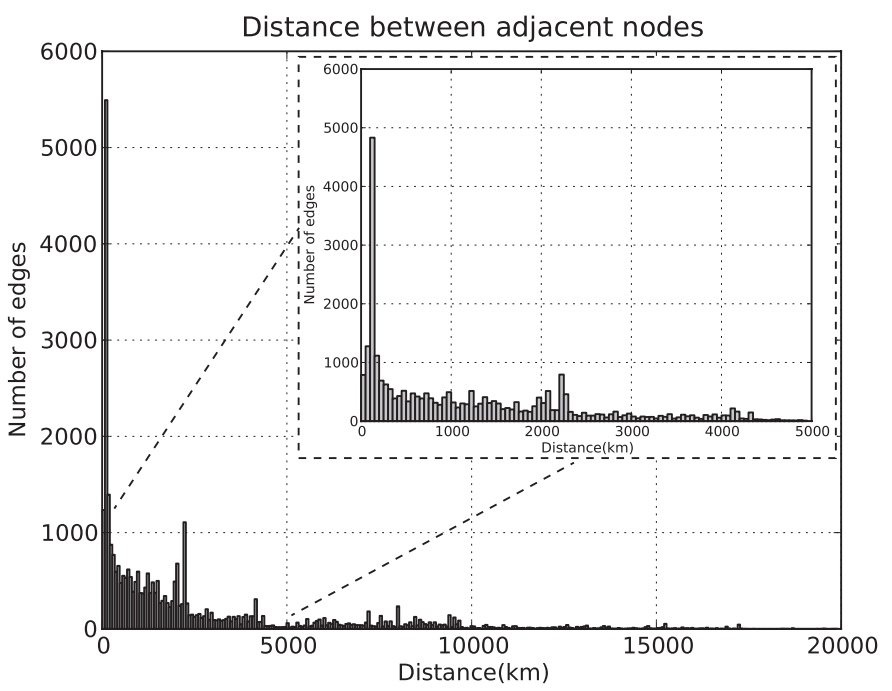

Fig. 7. Histogram of the distances of the adjacent nodes in the CAIDA topology. For better visualization, the inset illustrates the histogram of the range $0-5000 \mathrm{Km}$.

TABLE I. MTTR AND MTBF VALUES FOR OAS AND WDM LINE SYSTEMS

\begin{tabular}{|l|cc|}
\hline & MTBF(hours) & MTTR(hours) \\
\hline Bidirectional OA & $5 \times 10^{5}$ & 24 \\
Bidirectional WDM Line system & $5 \times 10^{5}$ & 6 \\
\hline
\end{tabular}

parent with known location. First we calculate the surface that can be covered by the parent node. This can be calculated based on the locations of other adjacent nodes to the parent. We only consider adjacent nodes with the distance between 50 and $1000 \mathrm{Km}$. We assume that nodes within the range of $50 \mathrm{Km}$ cover the same area and above $1000 \mathrm{Km}$ are probably not connected directly. Having this in mind, the radius of the surface that the parent node can cover is calculated as half of the average distance of the considered neighbors. If we assume that the leaf nodes are distributed uniformly in the covered area by the parent, half of the calculated radius can be considered as the upper bound for the distance of a leaf node to the parent.

Note that in practice, ASes might be connected to each other through Internet Exchange Points (IXP), however, in an AS-level topology such as CAIDA, these ASes are considered to be adjacent (an edge in the graph). This is a limitation of the model, as it considers a direct physical link between adjacent nodes in the AS-level topology.

Figure 7 depicts the distribution of the distances calculated for the adjacent nodes in the CAIDA network. As we see, a large percentage of distances are in the range of 10 to $5000 \mathrm{Km}$. Due to the incompleteness of the databases and the limitation of the model, a few distances reach $20 \mathrm{~K} \mathrm{Km}$. The average of the calculated distances is $2461.23 \mathrm{Km}$.

Using the calculated distances and formula (5) in Section IV, we evaluated the availability of the links/lines in the CAIDA network and Figure 8 depicts the distribution of

TABle II. Cable Cut and MtbF values for Fiberoptic Cable.

\begin{tabular}{|c|cc|}
\hline & Cable Cut-CC (km) & MTTR(hours) \\
\hline Terrestrial Fiberoptic Cable & 450 & 24 \\
\hline
\end{tabular}




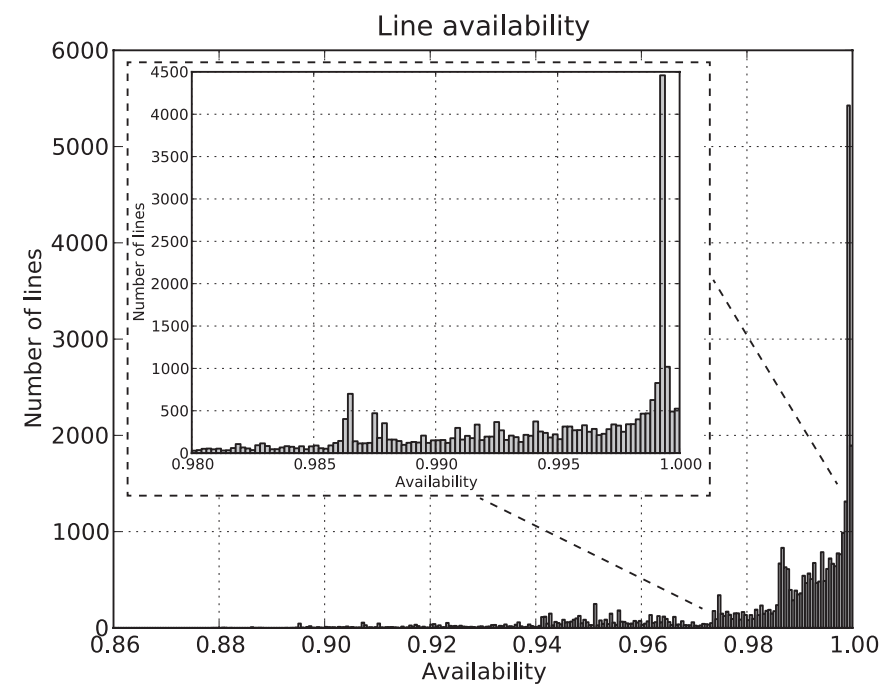

Fig. 8. Histogram of the line/link availability in the CAIDA topology. The inset illustrates the histogram of the range $0.98-1$.

TABLE III. PERCENTILE AND AVERAGE VALUES FOR LINE AVAILABILITY (A) IN THE CAIDA TOPOLOGY AND THE AVAILABILITY OF THE PROTECTED LINKS (A-P). (A-L) REPRESENTS THE AVAILABILITY FOR LINKS ATTACHED TO THE LEAF NODES.

\begin{tabular}{|l|cccccc|}
\hline & Min. & 25 & 50 & 75 & Max. & Average \\
\hline A & 0.8787 & 0.9820 & 0.9925 & 0.9986 & 0.99999 & 0.985 \\
A-P & 0.9852 & 0.99967 & 0.99994 & 0.99999 & 0.99999 & 0.9993 \\
A-L & 0.9852 & 0.99986 & 0.99999 & 0.99999 & 0.99999 & 0.99965 \\
\hline
\end{tabular}

the calculated values. The percentile and the average value of these availabilities are reported in Table III. The MTTR and MTBF of different optical components are based on the values proposed in [20] and are reported in Tables I and II. Considering the availability model of a bidirectional line (formula (5)), fiber optic cable is the dominant component in this model because the cable cuts are frequent and repair times are very long. Therefore, the availability of a line is dependent on its length. Observing the calculated line availabilities, the low minimum availability of 0.8787 can be explained because of the large distances $(20 \mathrm{~K} \mathrm{Km})$ between some nodes. Based on these results, up to 75 percentile of the line availabilities are below 0.999 .

Due to these large distances (low line availability) in order to have a realistic evaluation, the multi-layer model is considered in which links are assumed to be protected in lower layers (L2/L1). The percentile and the average value for availability of the protected lines are reported in Table III. We observe a significant enhancement in the availability of the lines compared to the values with no protection. The minimum line availability is increased to 0.9852 and even the 25 percentile of the values is above 0.999 .

\section{Numerical results}

Using the calculated line availabilities (with protection in lower layers L2/L1), we evaluate the connections availability using geometric routing in the CAIDA network based on formula (3) and the explanations in Section IV. The node availability is assumed to be 0.99994 which was proposed in [20].

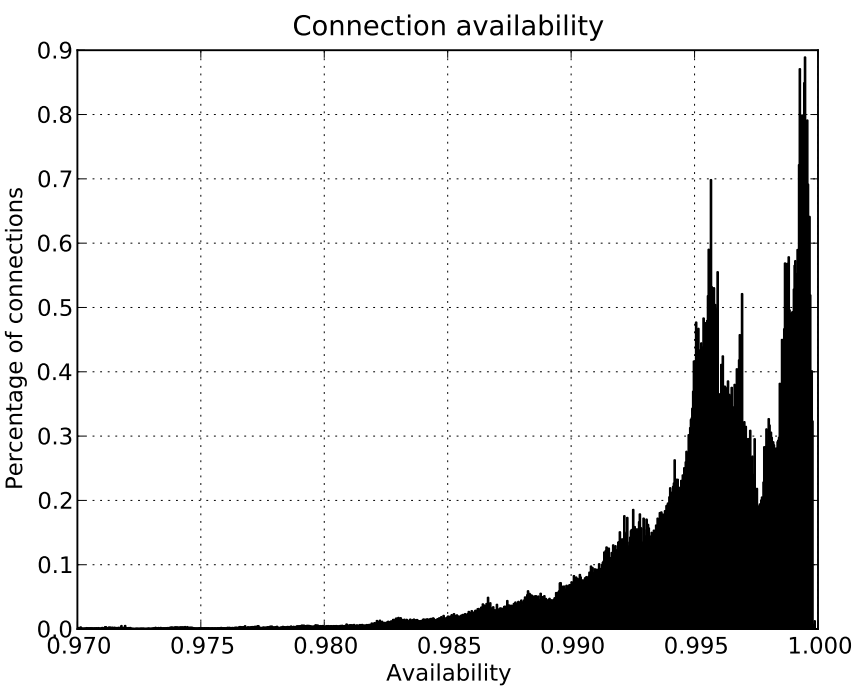

Fig. 9. Histogram of the connection availability of geometric routing on CAIDA topology without protection.

Note that in CAIDA topology, up to $30 \%$ of the nodes are leaf nodes. As these nodes/attached links can not be protected by the proposed protection scheme, their availability is limited to the obtained line availability with protection in lower layers (L1/L2). Table III reports the percentile of the availability of the edges attached to the leaf nodes. As the protection schemes do not enhance the availability of these edges, we report the availability of the connections excluding the leaves.

We first evaluate the connection availability without any recovery scheme and in the second experiment the protection scheme is considered and the explained simulation methodology is followed.

Figure 9 illustrates the distribution of the connections availability based on geometric routing in CAIDA network when no recovery is considered (for all possible source-destination pairs). For better visualization, the availability in the range 0.97-0.99985 is depicted however, the percentile and average values are reported in Table IV. As mentioned earlier, length of a link is the dominant factor in the availability of the link and the availability of a connection is also dependent on the length of the links. Therefore, availability of the connections including the very long links can be negatively affected. The low minimum value of 0.94202 can be explained because of the estimation of very large distances for some adjacent nodes in the network.

Figure 10 depicts the distribution of the connections availability with protection technique in geometric routing. We observe how the availability is enhanced compared to the previous scheme, when no recovery was used. The availability in the range 0.99980-0.99988 is depicted and the percentile and the average value are reported in Table IV. The minimum availability is increased to 0.98 and the average value is 0.9998 which is a significant enhancement compared to no protection scheme.

In the final scheme, as a comparison, we evaluate the connection availability in an alternative scheme in which the shortest cycle between every two nodes is calculated. Finding 


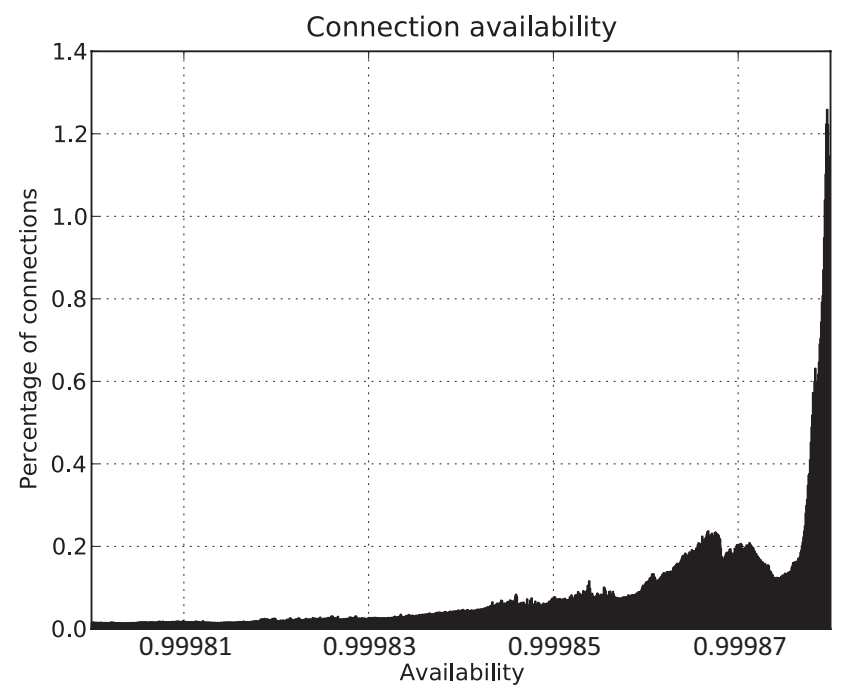

Fig. 10. Histogram of the connection availability of geometric routing on CAIDA topology with protection.

TABLE IV. PERCENTILE AND AVERAGE VALUES FOR CONNECTION AVAILABILITY IN 3 SCHEMES :I) GEOMETRIC ROUTING WITHOUT PROTECTION (G) II) GEOMETRIC ROUTING WITH PROTECTION (G-P) AND III) SHORTEST CYCLE (SH)

\begin{tabular}{|l|cccccc|}
\hline & Min & 25 & 50 & 75 & Max. & Average \\
\hline G & 0.94202 & 0.99388 & 0.99600 & 0.99845 & 0.99985 & 0.99531 \\
G-P & 0.98516 & 0.99984 & 0.99986 & 0.99987 & 0.99988 & 0.99983 \\
SH & 0.94841 & 0.99997 & 0.99999 & 0.99999 & 0.99999 & 0.99987 \\
\hline
\end{tabular}

the shortest cycle between two nodes in the topology boils down to finding minimum-cost flow of 2 between the nodes. Once the working path is unavailable, the second node-disjoint shortest path is used as the protection path. Availability of a connection is also dependent on the number of hop counts in that connection. The comparison with the shortest cycle scheme gives an overview on how the connection availability is affected by the increase in the number of hop counts. Figure 11 illustrates the distribution of the connections availability for the shortest cycle scheme. For better visualization, the values in the range of 0.9998 and 0.99999 are depicted. The percentile and the average values are reported in Table IV. An interesting observation is that, the minimum availability obtained by this scheme is much lower than the one achieved by the proposed protection scheme in geometric routing. This can be explained by the fact that for some connections in the topology, it might not be feasible to find two node disjoint paths while the proposed protection scheme is successful in finding an alternate path. The average connection availability achieved by both schemes are almost the same (0.9998). However, the maximum availability of the shortest cycle reaches 0.99999 while in the geometric scheme it reaches 0.99988 .

\section{SUMMARY AND FUTURE WORK}

We calculated connection availability of geometric routing over optical transport network on the Internet topology. Finding a reasonable model for the Internet topology was a challenge due to the incompleteness of the available data. This was tackled by using different databases and some estimations on the geolocation of the nodes in the Internet graph.

As fiber optic cable is the dominant component in the

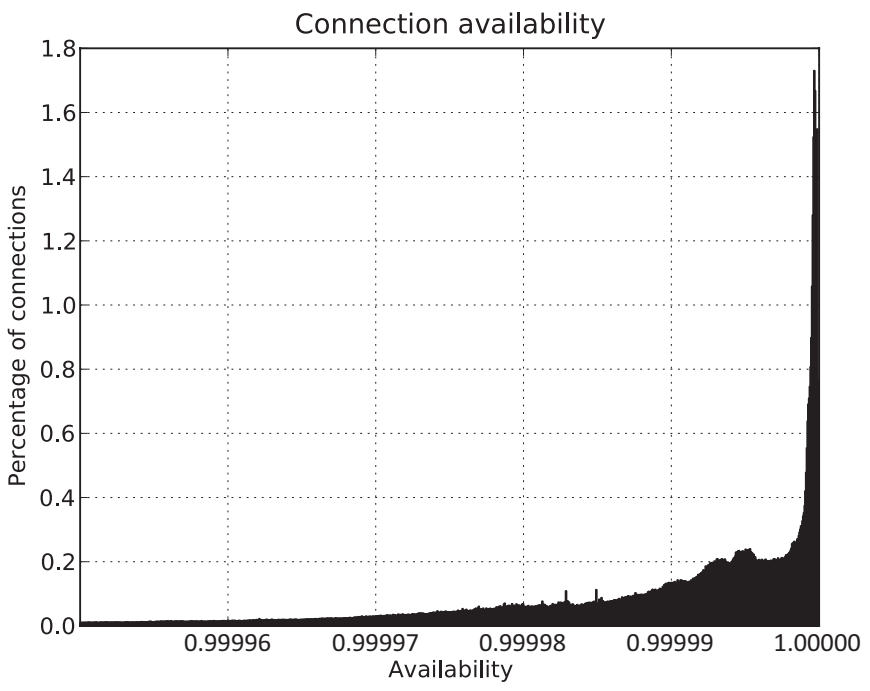

Fig. 11. Histogram of the connection availability of shortest cycle scheme on CAIDA topology.

availability model of network (frequency of the cuts and long repair times), the availability of connections is dependent on the distances of the adjacent nodes in the topology. Using the explained Internet model, the length of the links in the Internet topology ranges from $10 \mathrm{~km}$ to $20 \mathrm{~K} \mathrm{~km}$. Therefore, the availability of connections including the links with large length as $20 \mathrm{~K} \mathrm{~km}$ are considerably negatively affected. The other dominant factor in the connection availability is the hop counts between source and destination of the connections. However, the results in our prior work showed that the proposed protection schemes result into paths with length close to the shortest path length. The experimental results showed that the proposed scheme performs reasonably well compared to the shortest cycle alternative. Although the maximum achievable connection availability is higher in the shortest cycles, the minimum availability achieved by the proposed protection is much higher than the other scheme.

In large topologies such as Internet, the probability of multiple failures is not negligible. In order to obtain higher connection availability, protection against more failures should be considered. An interesting direction for future work is to evaluate the connection availability in geometric routing for multiple failure scenarios. Using recovery schemes against multiple failures should allow for high availability of up to 0.99999 as the ultimate goal.

\section{CONCLUSION}

In this paper, we considered geometric routing as an alternative to BGP to route between Autonomous Systems (ASes) in the Internet. We used a simple and promising greedy embedding based on a spanning tree of the network. As failures in the network might affect the greedy embedding, recovery techniques were considered to avoid packets reaching a dead end. We performed an availability analysis of the protection scheme for single failure in geometric routing on the Internet topology. For this analysis, we used a network model for the Internet topology which was challenging to achieve due to the incompleteness of the existing data. The availability was 
evaluated on a AS-level graph of CAIDA. In order to achieve a realistic evaluation, we used a multi-layer model in which links are protected in lower layers than network layer. The results showed significant enhancement in the availability compared to the scheme when no protection for the geometric routing was considered. We compared our results with an existing alternative in which the shortest cycle between the source and the destination is calculated. An interesting observation was that the minimum connection availability achieved by the proposed scheme was much higher than the shortest cycle scheme and the average connection availability obtained by both schemes were almost the same.

\section{ACKNOWLEDGMENT}

This work is partly funded by the European Commission through the EULER project (Grant 258307), part of the Future Internet Research and Experimentation (FIRE) objective of the Seventh Framework Programme (FP7).

\section{REFERENCES}

[1] G. Huston, "BGP routing table reports," 2013, http://bgp.potaroo.net/.

[2] R. Fonseca, S. Ratnasamy, J. Zhao, C. Ee, D. Culler, S. Shenker, and I. Stoica, "Beacon vector routing: Scalable point-to-point routing in wireless sensornets," in Proceedings of the 2nd conference on Symposium on Networked Systems Design \& Implementation-Volume 2. USENIX Association, 2005, pp. 329-342.

[3] C. Papadimitriou and D. Ratajczak, "On a conjecture related to geometric routing," Theoretical Computer Science, vol. 344, no. 1, pp. 3-14, 2005

[4] S. Sahhaf, W. Tavernier, D. Colle, M. Pickavet, and P. Demeester, "Single failure resiliency in greedy routing," in Proceedings of the 9th international conference on Design of Reliable Communication Networks, 2013, pp. 312-319.

[5] —, "Link failure recovery technique for greedy routing in the hyperbolic plane," Computer Communications, 2012.

[6] "The caida as relationships dataset," January 2004, http://www.caida.org/data/active/as-relationships/.

[7] M. Clouqueur and W. D. Grover, "Availability analysis of spanrestorable mesh networks," Selected Areas in Communications, IEEE Journal on, vol. 20, no. 4, pp. 810-821, 2002.

[8] D. Arci, G. Maier, A. Pattavina, D. Petecchi, and M. Tornatore, "Availability models for protection techniques in wdm networks," in Design of Reliable Communication Networks, 2003.(DRCN 2003). Proceedings. Fourth International Workshop on. IEEE, 2003, pp. 158-166.

[9] Q. She, X. Huang, and J. P. Jue, "How reliable can two-path protection be?" Networking, IEEE/ACM Transactions on, vol. 18, no. 3, pp. 922933, 2010.

[10] D. A. Mello, D. A. Schupke, and H. Waldman, "A matrix-based analytical approach to connection unavailability estimation in shared backup path protection," Communications Letters, IEEE, vol. 9, no. 9, pp. 844-846, 2005.

[11] J. Ahmed, C. Cavdar, P. Monti, and L. Wosinska, "Hybrid survivability schemes achieving high connection availability with a reduced amount of backup resources [invited]," Journal of Optical Communications and Networking, vol. 5, no. 10, pp. A152-A161, 2013.

[12] M. Azim and M. Kabir, "Availability study of M:N automatic protection switching scheme in WDM networks," Journal of High Speed Networks, vol. 18 , no. 1 , pp. $1-13,2011$

[13] —, "Availability analysis under multiple link failures in WDM networks with shared-link connections," Photonic Network Communications, vol. 23, no. 1, pp. 83-91, 2012.

[14] E. Calle, J. Segovia, and P. Vila, "Availability analysis of GMPLS connections based on physical network topology," Other IFIP Publications, no. $1,2011$.
[15] R. Kleinberg, "Geographic routing using hyperbolic space," in INFOCOM 2007. 26th IEEE International Conference on Computer Communications. IEEE, 2007, pp. 1902-1909.

[16] D. Eppstein and M. Goodrich, "Succinct greedy geometric routing using hyperbolic geometry," IEEE Transactions on Computers, vol. 60, no. 11, pp. 1571-1580, 2011.

[17] R. Flury, S. Pemmaraju, and R. Wattenhofer, "Greedy routing with bounded stretch," in INFOCOM 2009, IEEE, 2009, pp. 1737-1745.

[18] D. Chen and P. Varshney, "A survey of void handling techniques for geographic routing in wireless networks," IEEE Communications Surveys and Tutorials, vol. 9, no. 1, pp. 50-67, 2007.

[19] A. Cvetkovski and M. Crovella, "Hyperbolic embedding and routing for dynamic graphs," in INFOCOM 2009, IEEE, 2009, pp. 1647-1655.

[20] J. Vasseur, M. Pickavet, and P. Demeester, Network recovery: Protection and Restoration of Optical, SONET-SDH, IP, and MPLS. Morgan Kaufmann Publishers, 2004. 